\title{
EUS-guided Gastroenterostomy After Disuse of Enteric Metal Stents for Malignant Gastric Outlet Obstruction
}

\author{
Yonghua Shen ${ }^{1, \#}$, Lin Zhou ${ }^{1, \#}$, Xiaoping Zou ${ }^{1}$, Zhan Zhang ${ }^{2}$, Ying Lv ${ }^{1}$, \\ Zhenqing $\mathrm{Liu}^{3}$, Wen $\mathrm{Li}^{1}$, Guifang $\mathrm{Xu}^{1 *}$ and Lei Wang ${ }^{1 *}$ \\ ${ }^{1}$ Department of Gastroenterology, The Affiliated Drum Tower Hospital of Nanjing University, Medical School, Nanjing, P.R. China; \\ ${ }^{2}$ Department of Hygiene Analysis and Detection, School of Public Health, Nanjing Medical University, Nanjing, P.R. China; \\ ${ }^{3}$ Division of Oral Biology and Medicine, School of Dentistry, UCLA, 10833 Le Conte Ave, Los Angeles, USA; \\ \#These authors contributed equally to this work.
}

\begin{abstract}
Endoscopic ultrasound-guided gastroenterostomy (EUS-GE) appears to be suitable for malignant gastric outlet obstruction (GOO), serving as a hybrid treatment that includes the advantages of a long unobstructed period and less invasiveness. Two patients each, described in this report, had an unresectable cancer and underwent endoscopic metal stent placement in the stenotic enteric segment of a malignant GOO, which resulted in frequent stent replacement. EUS-GE was then performed with a fully covered, self-expandable, lumen-apposing metal stent, which combined a cautery tip-equipped access catheter. The patients' symptoms of nausea and vomiting were rapidly alleviated and did not recur during the follow-up period of 6 months. EUS-GE appears to be feasible after obstruction of a metal stent in a tumor segment. It may be a preferred alternative in patients with malignant GOO.
\end{abstract}

\section{Introduction}

Gastric outlet obstruction is a common problem in the terminal stage of many digestive malignancies, such as gastric cancer, duodenal cancer, periampullary cancer and pancreatic cancer. ${ }^{1}$ Gastric outlet obstruction (GOO) significantly reduces the patient's quality of life because it is often connected with symptoms of nausea, vomiting, abdominal distension, and loss of appetite. ${ }^{2}$

In recent years, endoscopic enteric metal stent placement for the treatment of malignant GOO has become one of the alternatives to surgical gastrojejunostomy, providing the benefit of being less invasive. ${ }^{3,4}$ However, recurrent obstructive symptoms were common after stent placement because of cancer tissue in-growth. ${ }^{5}$ Endoscopic ultrasound-guided gastroenterostomy (EUS-GE) appears to be more suitable for malignant GOO, serving as a hybrid treatment that includes both advantages of a long unobstruction period in surgical gastrojejunostomy and less invasiveness in endoscopic

Keywords: Disuse; Enteric metal stents; EUS-guided gastroenterostomy; Malignant gastric outlet obstruction.

Abbreviations: EUS-GE, endoscopic ultrasound-guided gastroenterostomy; GOO, gastric outlet obstruction; LAMS, lumen-apposing metal stent.

Received: May 14, 2019; Revised: May 28, 2019; Accepted: May 30, 2019

${ }^{*}$ Correspondence to: Guifang Xu and Lei Wang, Department of Gastroenterology, The Affiliated Drum Tower Hospital of Nanjing University, Medical School, Nanjing, P.R. China. E-mail: 13852293376@163; 867152094@qq.com

How to cite this article: Shen Y, Zhou L, Zou X, Zhang Z, Lv Y, Liu Z, Li W, Xu G, Wang L. EUS-guided Gastroenterostomy After Disuse of Enteric Metal Stents for Malignant Gastric Outlet Obstruction. Exploratory Research and Hypothesis in Medicine 2019;4(3):62-64. doi: 10.14218/ERHM.2019.00011. metal stent placement. ${ }^{6}$

Hence, we present herein two EUS-GE cases to support the successful use of this procedure as an alternative treatment to enteric stent placement.

\section{Case report}

The two patients each had an unresectable cancer and underwent endoscopic metal stent placement in the stenotic enteric segment of malignant GOO (one patient underwent the procedure once and the other three times). One patient was a 45 -year-old female who suffered from cholangiocarcinoma and had received radiotherapy and chemotherapy prior to the EUS-GE. The other patient was a 53-year-old male who suffered from gastric adenocarcinoma. Both patients provided informed consent for publication of this case. The case report was also approved by the local research ethics committee.

The average gastric outlet maintenance period for these two patients was 102 days, and this resulted in frequent stent replacement (Fig. 1a). EUS-GE was performed with a fully covered, selfexpandable, lumen-apposing metal stent (LAMS) (12 mm in diameter and $2.5 \mathrm{~cm}$ in length), which combined a cautery tip-equipped access catheter (MicroTech, Nanjing, China). The LAMS was then deployed between the stomach and jejunum without any adverse event (Fig. 1b; Supplementary Video 1). A gastroscope (external diameter $8.9 \mathrm{~mm}$ ) was able to pass through the stent into the jejunum (Fig. 1c). The average total procedure time was 68.5 min (68 and 69 min respectively). The average time between puncture 


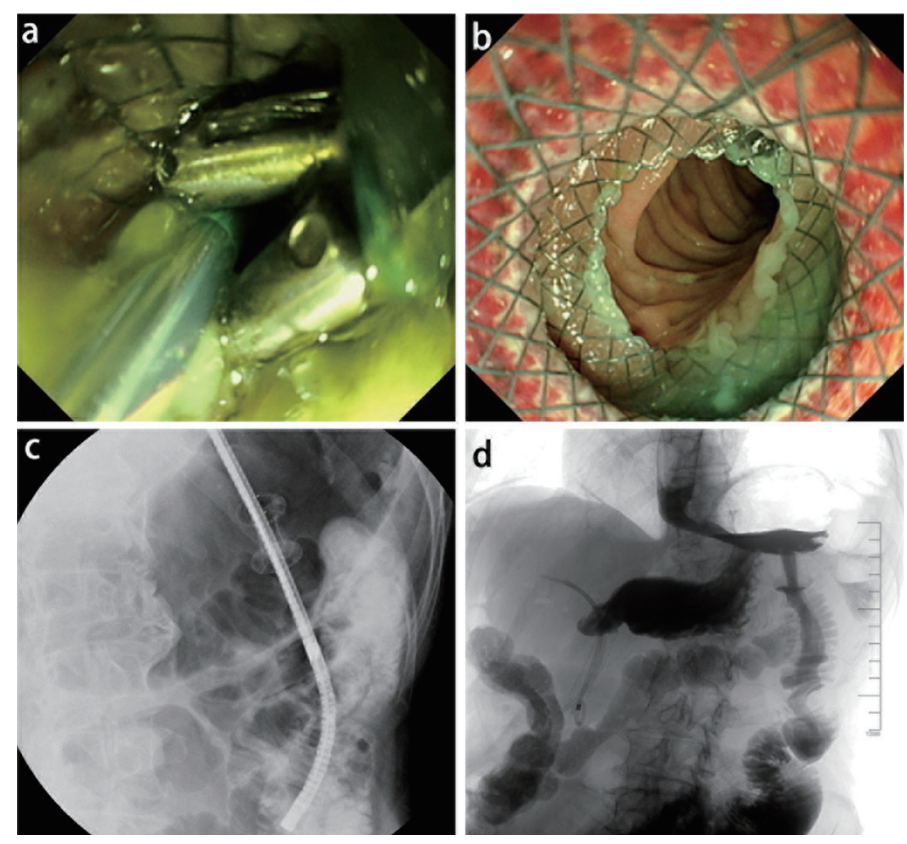

Fig. 1. Endoscopic ultrasound-guided gastroenterostomy after disuse of a metal stent for malignant gastric outlet obstruction. a) Obstruction of metal stent for malignant gastric outlet obstruction. b) Proximal flange was deployed under endoscopic imaging. c) Fluoroscopic view of the gastroscope being passed through the stent into the jejunum. d) The follow-up upper gastrointestinal examination with gastrografin showed contrast medium flowing well from stomach to the jejunum.

and successful delivery of the stent was $42.5 \mathrm{~min}$ (40 and $45 \mathrm{~min}$ respectively). All procedures were performed by two expert endoscopists.

There was no complication in either of the two patients. The two patients reported feeling slight epigastric pain which remitted the next day. The patients' symptoms of nausea and vomiting rapidly disappeared. Upper gastrointestinal examination with gastrografin on the following day showed the stent to be functioning well (Fig. 1d). The symptoms did not recur during the follow-up of 6 months.

\section{Discussion}

Surgical gastrointestinal anastomosis is a traditional treatment for $\mathrm{GOO}$, but it requires patients to be in good condition. Endoscopic metal stent placement in the stricture area is relatively simple and less traumatic, but stent clogging requires repeated implantation when the cause of stenosis is malignant because of the rapid growth of tumor tissue into the stent, which brings more pain to the patients. Recently, EUS-GE has become an alternative treatment. It uses a LAMS to establish a new pathway between the stomach and jejunum, thus bypassing the stricture site. It has the advantages of minimal invasion, being a nonblocking stent, and maintaining patency for a long time. A number of studies have shown that EUS-GE is safe and effective, with a technical success rate and a clinical success rate of $87-90 \%{ }^{7,8}$ Compared to enteral stent placement, EUS-GE has a higher initial clinical success rate and lower stent failure rate requiring repeat intervention. ${ }^{9}$

The main complications of EUS-GE include intestinal fistula, bleeding, and stent displacement. The emergence of a new type of LAMS significantly reduced the occurrence of stent displacement in different endoscopic anastomosis procedures. In EUS-GE, a number of studies have taken the lead in the application of LAMS in animal experiments, without occurrence of stent displacement. ${ }^{6}$ Since then, this type of stent has been successfully used in a number of human patients who have undergone this technique. The emergence of an intestinal fistula is mainly related to the operation process itself. Gastrointestinal perforation can be produced during the puncture. However, as long as stents can be successfully placed, the design of LAMS can block the perforation site and effectively prevent further intestinal fistula.

The experiences with our cases indicate that EUS-GE can be safe and effective after disuse of enteric metal stents for malignant GOO. However, the EUS-GE procedure should be performed by expert endoscopists. EUS-GE is a promising treatment for malignant GOO and more patients will benefit from it.

\section{Conclusions}

EUS-GE appears to be feasible after obstruction of a metal stent in a tumor segment. Further, it may be a preferred alternative in malignant GOO patients.

\section{Conflict of interest}

The authors have no conflict of interests related to this publication.

\section{Author contributions}

Designing the research (LW, GFX); performing the research (YHS, LZ, ZZ, YL, ZQL, XPZ and WL); collecting the data (YHS, LZ and GFX); writing the manuscript (YHS), critically 
revising the manuscript for important intellectual content (LW, XPZ, GFX).

\section{Supporting information}

Supplementary material for this article is available at https://doi. org/10.14218/ERHM.2019.00011.

Supplementary Video 1. Endoscopic ultrasound-guided gastroenterostomy for the treatment of malignant gastric outlet obstruction.

\section{References}

[1] Koop AH, Palmer WC, Stancampiano FF. Gastric outlet obstruction: A red flag, potentially manageable. Cleve Clin J Med 2019;86:345-353. doi:10.3949/ccjm.86a.18035.

[2] Potz BA, Miner TJ. Surgical palliation of gastric outlet obstruction in advanced malignancy. World J Gastrointest Surg 2016;8:545-555. doi:10.4240/wjgs.v8.i8.545.

[3] Maetani I, Tada T, Ukita T Inoue H, Sakai Y, Nagao J. Comparison of duodenal stent placement with surgical gastrojejunostomy for palliation in patients with duodenal obstructions caused by pancrea- ticobiliary malignancies. Endoscopy 2004;36:73-78. doi:10.1055 /s-2004-814123.

[4] Minata MK, Bernardo WM, Rocha RS, Morita FH, Aquino JC, Cheng S, et al. Stents and surgical interventions in the palliation of gastric outlet obstruction: a systematic review. Endosc Int Open 2016;4:E1158E1170. doi:10.1055/s-0042-115935.

[5] Jeurnink SM, van Eijck CH, Steyerberg EW, Kuipers EJ, Siersema PD. Stent versus gastrojejunostomy for the palliation of gastric outlet obstruction: a systematic review. BMC Gastroenterol 2007;7:18. doi:10.1186/1471-230X-7-18.

[6] Binmoeller KF, Shah JN. Endoscopic ultrasound-guided gastroenterostomy using novel tools designed for transluminal therapy: a porcine study. Endoscopy 2012;44:499-503. doi:10.1055/s-0032-1309382.

[7] Khashab MA, Kumbhari V, Grimm IS, Ngamruengphong S, Aguila G, El Zein $M$, et al. EUS-guided gastroenterostomy: the first U.S. clinical experience (with video). Gastrointest Endosc 2015;82:932-938. doi:10.1016/j.gie.2015.06.017.

[8] Khashab MA, Bukhari M, Baron TH, Nieto J, El Zein M, Chen YI, et al. International multicenter comparative trial of endoscopic ultrasonography-guided gastroenterostomy versus surgical gastrojejunostomy for the treatment of malignant gastric outlet obstruction. Endosc Int Open 2017;5(4):E275-E281. doi:10.1055/s-0043-101695.

[9] Ge PS, Young JY, Dong W, Thompson CC. EUS-guided gastroenterostomy versus enteral stent placement for palliation of malignant gastric outlet obstruction. Surg Endosc 2019, [Epub ahead of print] doi:10.1007/s00464-018-06636-3. 